\title{
BioéthiqueOnline
}

\section{Cobayes de la relève : l'artiste et le chercheur à la rencontre de} l'expérience

\section{Jean-Frédéric Ménard}

Volume 5, 2016

URI : https://id.erudit.org/iderudit/1044276ar

DOI : https://doi.org/10.7202/1044276ar

Aller au sommaire du numéro

Éditeur(s)

BioéthiqueOnline

ISSN

1923-2799 (numérique)

Découvrir la revue

Citer cet article

Ménard, J.-F. (2016). Cobayes de la relève : l'artiste et le chercheur à la rencontre de l'expérience. BioéthiqueOnline, 5.

https://doi.org/10.7202/1044276ar
Résumé de l'article

Cet essai se veut une réflexion préliminaire dans la foulée de la collaboration amorcée avec une artiste, Arkadi Lavoie Lachapelle, dans le cadre d'un projet interdisciplinaire sur l'art et la bioéthique. Je dirai d'abord quelques mots à propos de la conception du droit à laquelle j'adhère et des conclusions méthodologiques que j'en tire. Ces remarques serviront d'arrière-plan aux réflexions qui m’ont été inspirées par ma collaboration avec Arkadi Lavoie Lachapelle dans le cadre de ce projet. Ensuite, je m'aventurerai du côté de la critique artistique en proposant l'esquisse d'une lecture de certaines oeuvres de l'artiste. Enfin, je ferai quelques remarques réflexives touchant à mon expérience éthique de la pratique d'une méthodologie de recherche qualitative et aux rapprochements qui peuvent être faits avec la pratique artistique. 


\title{
Cobayes de la relève : l'artiste et le chercheur à la rencontre de l'expérience
}

\author{
ESSAI / ESSAY \\ Jean-Frédéric Ménard ${ }^{1,2}$
}

\author{
Reçu/Received: 20 Feb $2016 \quad$ Publié/Published: 16 Sept 2016 \\ Éditeurs/Editors: Jean-Christophe Belisle-Pipon \& Renaud Boulanger \\ Travail créatif/Creative work: Lavoie-Lachapelle A. Jour de fête! BioéthiqueOnline. 2016;5/17
}

2016 J-F Ménard, Creative Commons Attribution 4.0 International License

\section{Résumé}

Cet essai se veut une réflexion préliminaire dans la foulée de la collaboration amorcée avec une artiste, Arkadi Lavoie Lachapelle, dans le cadre d'un projet interdisciplinaire sur l'art et la bioéthique. Je dirai d'abord quelques mots à propos de la conception du droit à laquelle j'adhère et des conclusions méthodologiques que j'en tire. Ces remarques serviront d'arrière-plan aux réflexions qui m'ont été inspirées par ma collaboration avec Arkadi Lavoie Lachapelle dans le cadre de ce projet. Ensuite, je m'aventurerai du côté de la critique artistique en proposant l'esquisse d'une lecture de certaines œuvres de l'artiste. Enfin, je ferai quelques remarques réflexives touchant à mon expérience éthique de la pratique d'une méthodologie de recherche qualitative et aux rapprochements qui peuvent être faits avec la pratique artistique.

\section{Mots clés}

réflexivité, art, éthique de la recherche, droit, recherche qualitative, interdisciplinarité, performance

\section{Summary}

This essay is a preliminary reflection in the wake of the collaboration initiated with an artist, Arkadi Lavoie Lachapelle, as part of an interdisciplinary project on art and bioethics. I will first say a few words about the concept of law to which I adhere and the methodological conclusions that I draw. These remarks will serve as background to the reflections that were inspired by my collaboration with Arkadi Lavoie Lachapelle in this project. I will then venture to the side of art criticism by proposing an outline of a reading of certain works of the artist. Finally, I will make a few remarks related to my reflexive ethical experience of the practice of qualitative research methodology and comparisons that can be made with artistic practice.

\section{Keywords}

reflexivity, research ethics, law, qualitative research, interdisciplinarity, performance

\section{Affiliations des auteurs / Author Affiliations \\ ${ }^{1}$ Faculty of Laws, University College London, Londres, Angleterre \\ ${ }^{2}$ Centre d'éthique appliquée, Centre universitaire de santé McGill, Montréal, Canada}

\section{Correspondance / Correspondence}

Jean-Frédéric Ménard, j.menard.12@ucl.ac.uk

\section{Remerciements}

Avant tout, je tiens à remercier Arkadi Lavoie Lachapelle d'avoir joué le jeu de cette improbable rencontre entre une artiste et un bioéthicien avec autant de sérieux, d'entrain et d'ouverture ainsi que pour ces commentaires sur une version antérieure de ce texte. Je remercie aussi Elsa Laflamme pour sa lecture attentive et ses commentaires. Je suis aussi redevable aux éditeurs de BioéthiqueOnline pour leurs judicieuses observations. Enfin, merci aux organisateurs et organisatrices du projet Art + Bioéthique de nous avoir donné cette formidable opportunité.

\section{Conflit d'intérêts}

Aucun déclaré

\section{Acknowledgements}

Above all, I thank Arkadi Lavoie Lachapelle for having played the game of this unlikely encounter between an artist and a bioethicist in a serious, enthusiastic and open manner, as well as the comments on an earlier version of the text. I thank Elsa Laflamme for her careful reading and comments. I am also indebted to the editors of BioéthiqueOnline for their insightful comments. Finally, thanks to the organizers of the project Art + Bioéthique for giving us this wonderful opportunity.

\section{Conflicts of Interest}

None declared

\section{Introduction}

Après avoir eu le plaisir et l'honneur de collaborer et d'échanger avec Arkadi Lavoie Lachapelle dans le cadre du projet qui donne lieu à ce dossier, je suis aux prises avec un foisonnement d'idées et d'intuitions nouvelles auquel il serait difficile de rendre justice dans un court texte. Néanmoins, je 
tenterai de poser quelques jalons qui marqueront le chemin parcouru, mais surtout indiqueront celui qui se dessine à l'horizon.

Au-delà de tout ce qui peut éloigner une artiste contemporaine et un chercheur universitaire, il y a de profondes ressemblances, tant sur le plan des thèmes abordés que sur celui de l'approche. Mes recherches ont l'éthique comme " objet » puisque je m'intéresse à l'émergence et à la négociation des normes encadrant la prise de décision éthique et juridique aux soins intensifs néonataux. De la même façon, je décèle dans le travail d'Arkadi Lavoie Lachapelle le désir de susciter un questionnement sur les conventions sociales, que ce soit l'institution muséale, notre rapport à la naissance ou notre conception de l'espace public. Ensuite, et c'est peut-être un parallèle encore plus fort que le précédent, nous partageons ce désir d'aller vers l'expérience, de l'interroger et ultimement de la transformer.

J'aborderai cette réflexion en trois temps. Tout d'abord, je dirai quelques mots à propos de la conception du droit à laquelle j'adhère et des conclusions méthodologiques que j'en tire. Ces quelques remarques initiales serviront ensuite d'arrière-plan aux réflexions qui m'ont été inspirées par ma collaboration avec Arkadi Lavoie Lachapelle dans le cadre de ce projet. Ensuite, je m'aventurerai du côté de la critique artistique en proposant l'esquisse d'une lecture des œuvres de l'artiste dont nous avons eu l'occasion de discuter. Enfin, je reviendrai en terrain plus connu pour faire quelques remarques réflexives touchant à mon expérience éthique de la pratique d'une méthodologie de recherche qualitative et aux rapprochements qui peuvent être faits avec la pratique artistique.

\section{L'expérience et la recherche en droit}

Le droit intervient directement dans le réel. Sans être le seul, il est un élément constitutif de notre expérience subjective et sociale. D'une situation donnée, il permet de construire ce que Robert Cover appelle une " alternité » [1] (je traduis le néologisme qu'il emprunte à George Steiner combinant " alternative » et « reality »). Ainsi, le droit pose un idéal, il est performatif et normatif, en cela qu'il permet de passer d'une situation telle qu'elle est à telle que nous croyons qu'elle devrait être.

Le changement peut-être quasi instantané : prenons l'exemple de Simone et Marie qui cherchent un logis et signent un contrat de bail d'habitation avec Annie. Le rapport entre ces trois personnes vient de changer, ce qui a aussi pour effet de redéfinir leur rapport à ce construit - à la fois au sens matériel du plâtre et de la brique et au sens social d'un réseau de concepts et d'attentes - que l'on appelle un appartement. Ce serait une erreur de croire toutefois que le droit formel et étatique, dans ce cas le contrat de bail, fait table rase et occupe tout l'espace normatif. Si elles peuvent être transformées par le contrat, les relations préexistantes et à venir entre Simone, Marie et Annie ne disparaissent pas. Peut-être Marie et Annie sont-elles cousines, cette dernière consentant un loyer avantageux par souci de donner un coup de pouce à sa cousine. Peut-être Annie a-t-elle eu à faire face à plusieurs plaintes concernant de bruyants anciens locataires et le strict avertissement qu'elle sert à Annie et Marie à ce sujet peut avoir à ses yeux une bien plus grande importance que n'importe quel article du bail. De même, toutes trois demeurent libres de définir leurs relations les unes avec les autres de façon informelle, sans nécessairement référer au texte du contrat de bail. Celui-ci vient néanmoins d'entrer dans leurs vies et à n'importe quel moment, n'importe laquelle des trois parties au contrat pourra y avoir recours pour obtenir quelque chose, que ce soit le paiement d'un loyer en retard ou une réparation.

Bien entendu, on peut aussi « changer le monde " à plus grande échelle, tant dans l'espace que dans le temps. L'émergence des droits de la personne comme catégorie pour penser le rapport des individus entre eux et avec les différentes instances gouvernementales auxquelles ils sont assujettis constitue à cet égard un exemple éloquent. Pour n'évoquer qu'un seul exemple étroitement lié aux débats "classiques " de la bioéthique, pensons à la question de l'aide médicale à mourir et du suicide assisté. De la stricte prohibition du suicide dans le Code criminel canadien, abolie en 1972, 
aux revendications de Sue Rodriguez [2] jusqu'à la décision récente de la Cour suprême du Canada dans l'arrêt Carter [3], où l'expertise des sciences sociales a eu tellement de poids, on doit comprendre que le droit ne fonctionne pas en circuit fermé. Autant le droit peut-il contribuer à l'évolution des mœurs, autant est-il perméable aux transformations sociétales.

En effet, peu importe l'échelle à laquelle on se place, le droit, comme l'écrit Emmanuel Melissaris, repose sur la rencontre des attentes normatives partagées par les membres d'une communauté donnée [4]. Comme ces attentes sont susceptibles de changer au gré des interactions sociales, le droit n'est donc pas statique et immuable et ne peut être réduit ni aux décisions des juges, ni aux textes de loi, ni aux interprétations savantes des unes ou des autres. C'est aussi dans cette optique que je juge qu'il est intellectuellement plus productif de faire des rapprochements entre le droit (au sens restreint de droit étatique), l'éthique et la morale et de les placer sur un continuum plutôt que de s'efforcer d'en décliner toutes les différences à l'instar du positivisme juridique.

Voilà donc pourquoi, en tant que chercheur aux identités multiples - un peu philosophe, juriste et bioéthicien -, je m'intéresse aux méthodologies émergentes confrontant les analyses philosophiques et juridiques à l'épreuve de l'expérience vécue des individus qui font face à des questionnements se résumant à la grande question de l'éthique (et du droit) : comment faire pour bien faire? Ainsi, mon projet doctoral sur l'éthique des soins intensifs néonataux repose sur une proposition méthodologique adaptée que j'ai nommée " théorisation normative enracinée ». La théorisation normative enracinée s'inscrit dans le courant de l'éthique empirique [5] et fait une très large part à une enquête empirique qualitative inspirée de la théorisation enracinée [6]. En " allant à la rencontre de l'expérience » [7], j'espère être en mesure de mieux saisir les enjeux émergeant de la pratique et sur la base de cette compréhension, être en mesure d'appréhender comment ces derniers pourraient être résolu, en tout ou en partie.

\section{L'expérience dans l'art}

Tout comme j'aspire à saisir le droit au-delà des murs du Palais de justice et des recueils de jurisprudence, la pratique artistique d'Arkadi Lavoie Lachapelle ne se limite pas aux musées ou aux galeries d'art. De même, elle vise une réévaluation et une reconfiguration des rapports sociaux. Ainsi, en déposant en pleine nuit " 1600 œufs " [8], littéralement 1600 coquilles d'œufs remplies de paillettes dorées, sur le parvis du Musée d'art contemporain de Montréal, elle et sa collègue Audrey Racicot interrogeaient à la fois ce qui fait l'œuvre, ce qui fait l'institution muséale et ce qui fait l'artiste en court-circuitant le processus en vertu duquel le musée commande et défini les œuvres. Puis, dans la vidéo de 2015 «Parlons d'œufs ! » [9] qui fait suite à cette manœuvre clandestine de 2010, elle donne la parole, sans égard pour les hiérarchies, aux différents acteurs et actrices du musée, du portier jusqu'à la haute direction, pour recueillir leurs réactions et impressions. Ce faisant, elle suggère une esthétique décentralisée et démocratique où le rôle de commissaire et de critique d'art revient à celui qui se l'approprie plutôt qu'exclusivement à ceux et celles qui en sont institutionnellement investis.

De même, en 2011 dans " Jour de fête » [10], elle met en cause les notions d'espace public, de propriété privée, de liberté d'expression et de sécurité. Les interactions auxquelles donnent lieu l'arrivée au pied d'une tour de bureaux du centre des affaires de Montréal d'une femme offrant à la vue des personnes qui occupent les étages supérieurs un bouquet de ballons aux couleurs festives sont révélatrices des tensions éthico-politiques qui existent dans notre société entre l'espace public et l'espace privé, mais aussi de l'effectivité des attentes et des conceptions de ce qui est légal et de ce qui ne l'est pas que chaque individu porte en lui. En s'engageant dans un dialogue sincère, notamment avec les responsables de la sécurité de l'immeuble, l'artiste met en jeu autant ses propres préconceptions que celles de ses interlocuteurs pour négocier un ordre juridique et éthique qui répondent aux attentes et aux responsabilités de chacun. 
Dans cette œuvre, Arkadi Lavoie Lachapelle se met elle-même en scène dans une expérience sociale dont les résultats sont fascinants tant pour le juriste que pour le bioéthicien. Le chercheur universitaire qui voudrait étudier ces mêmes questions avec les mêmes moyens serait-il autorisé à le faire? D'une part, est-ce que les canons de la discipline reconnaitraient la valeur d'une telle approche? D'autre part, une telle intervention ne serait-elle pas considérée comme relevant de la recherche avec des sujets humains et, partant, assujettie à l'évaluation d'un comité d'éthique de la recherche?

Cet exemple est loin d'être le seul où des pratiques très similaires, voire identiques, sont sujettes à des cadres normatifs différents. Souvent, le contexte fait la différence. Ainsi deux individus échangeant de violents coups de poing au visage seront susceptibles d'être arrêtés et accusés de voies de fait ou d'avoir troublé la paix, sauf s'il s'agit d'un combat de boxe se déroulant selon les règles de ce sport et sous la supervision d'un arbitre ou d'un entraineur compétent.

De même, l'œuvre proposée par Arkadi Lavoie Lachapelle aux fins du présent projet de rencontre entre l'art et la bioéthique fait progresser cette réflexion dans le travail de l'artiste. En effet, « Jour de fête! » (notons le point d'exclamation qui la distingue de la performance de 2011) est l'aboutissement d'une succession de changement de cadre, tant au plan matériel que symbolique. La photo prise par Isabelle Brabant est susceptible de donner lieu à plusieurs interprétations; elle documente en même temps la naissance d'un enfant, l'accouchement d'une mère et le travail du médecin. Elle témoigne d'une époque, d'une approche de la naissance et de l'accouchement. Elle marque le passage, l'acquisition imminente du statut de mère pour la femme qui accouche et celui de personne pour l'enfant. Elle représente peut-être le souvenir chéri d'un jour heureux, ou le rappel de l'immense douleur d'une naissance difficile. Dans l'éventualité d'un conflit entourant la naissance, elle aura même pu être soumise à l'appréciation d'un ou d'une juge, questionnée comme un témoignage.

Par son cadrage, la photo parle autant qu'elle tait. Elle place l'enfant au centre de l'image, reflétant par-là la priorité du meilleur intérêt de celui-ci sur celui des parents, dans ce cas plus spécifiquement la mère, ou celui du corps médical. Or, cet enfant est-il même déjà né aux yeux de la société représentée par le droit? Cet enfant est-il déjà sujet de droit et débiteur d'obligations de la part des autres acteurs qui se trouvent dans l'image. Est-il encore " en ventre sa mère " comme le dirait le common lawyer? Est-il né vivant et viable tel que demanderait le civiliste? Toutes ces questions sont suggérées et inscrites dans la photo.

En se saisissant de cette photographie pour en faire œuvre, l'artiste opère un changement de cadre normatif. Les règles applicables tant à son utilisation qu'à son interprétation ne sont plus tout à fait les mêmes. En l'agrandissant et en lui donnant un encadrement matériel, elle détache cette scène inscrite dans l'histoire particulière d'une naissance pour en faire une scène aux échos beaucoup plus généraux. En un sens, ce n'est plus tant de la naissance de cet enfant et de l'accouchement de cette mère dont il est question que de l'incarnation d'une certaine expérience de la naissance et de l'accouchement dans le contexte médical occidental. Un « universel concret » aurait pu dire Hegel.

En renversant la photographie pour en faire œuvre, Arkadi Lavoie Lachapelle introduit une distance supplémentaire, ajoutant à la distance de la photographe celle du spectateur. Elle appelle l'interprétation et met en cause l'ordre établi et les relations de pouvoir qui sont sous-jacentes à la scène de la naissance. La dimension chimérique et paradoxale de la femme enceinte et parturiente ne s'appartenant plus est soulignée en faisant de la tête de l'enfant naissant celle du corps de sa mère. En mettant de l'avant le rôle de la sage-femme ayant pris le cliché dans le cadre de ses fonctions dans le cartel accompagnant l'œuvre, l'artiste nous permet également d'entrevoir au-delà de l'image les enjeux éthico-politiques entourant la naissance et le rôle respectif du médecin et de la sage-femme. 


\section{L'éthique dans l'art et dans la recherche qualitative}

Au-delà de l'éthique comme thème ou comme objet d'étude, l'art-action tel que le pratique Arkadi Lavoie Lachapelle et la recherche qualitative ayant pour but de susciter une rencontre avec l'expérience suscitent aussi leur lot de questionnements éthiques. Tant la pratique artistique d'Arkadi Lavoie Lachapelle que mes recherches doctorales impliquent des participants humains. Comme je l'ai évoqué d'entrée de jeu avec l'exemple du contrat de bail, à priori l'on pourrait croire que le formulaire de consentement que je présente aux personnes qui participent à mon projet et où sont détaillés leurs droits en tant que participants et mes engagements en tant que chercheur, constitue le fondement normatif de notre relation. Or, on ne s'y réfèrera que si quelque chose devait mal tourner. En ce sens, le droit formel et écrit ne sert que de filet de sécurité. Dans l'hypothèse la plus optimiste, il a vocation à être redondant. Lorsque je m'assois dans une salle avec une professionnelle de la santé pour discuter de son rapport à ses patients et aux questions éthico-juridiques que soulève son travail, je dois établir un lien de confiance avec cette personne qui, souvent, me parlera sans beaucoup de retenue de son expérience, de ses angoisses, de ses interrogations. Je suis responsable de respecter la confidence qui m'est faite. Je suis aussi responsable de donner une voix à ces personnes qui acceptent de collaborer avec moi à la réalisation de mon projet de recherche. Pour reprendre ce que j'ai dit à propos de "Jour de fête ", il nous faut négocier un ordre juridique et éthique qui réponde aux attentes et aux responsabilités de chacun.

Ainsi, les défis sont nombreux. D'une part, l'on espère dépasser ce qu'il peut y avoir de banal et d'unilatéral dans l'expérience d'une seule personne pour en saisir les ressorts et la logique. Par ailleurs, l'on cherche aussi à préserver l'éclairage unique qu'apporte une perspective qui, malgré les convergences des perspectives, ne sera jamais entièrement celle d'un autre individu. De plus, il y a un réel risque d'instrumentalisation de la contribution des participants dont il me parait crucial d'être conscient. À la force de l'argument ou de l'œuvre, il est possible de sacrifier l'intégrité d'un participant ou de le placer dans une position de vulnérabilité. Tant le chercheur que l'artiste doivent s'efforcer de ne pas verser dans ce que l'on pourrait nommer le « vampirisme de l'expérience ». Si leurs travaux et, par conséquent, leurs succès professionnels dépendent de l'apport d'autrui dont ils se nourrissent, pour agir éthiquement, artistes et chercheurs doivent s'assurer qu'ils ne s'emparent pas de toute la force vitale de leurs collaborateurs, qu'ils ne les laissent pas endommagés lorsque se termine leur interaction avec eux. À cet égard, pensons aux protagonistes mêmes du " Jour de fête! » d'Arkadi Lavoie Lachapelle : la mère, l'enfant et le médecin. Le rapprochement entre l'art et la bioéthique nous incite à nous demander ce qu'il en est du droit à l'image et à la vie privée de ces personnes qui, pour au moins deux d'entre elles, verront une partie intime de leur vie littéralement révélée au grand jour. Le propos général et métaphorique de l'œuvre tire une part de sa force dans l'intensité de la situation particulière qu'elle dépeint. Ainsi, à moins de choisir sciemment la voie de la transgression, l'artiste ne saurait s'approprier cette image et l'affecter à son œuvre sans avoir d'égards pour les intérêts des individus qui s'y trouvent engagés.

De même, au-delà des individus, j'ai aussi une responsabilité quant au milieu de vie que j'étudie et dans lequel je m'immisce. Bien que l'objectif de mes recherches est de mettre au jour les mécanismes et les processus sociaux qui sont à l'œuvre dans les unités de soins intensifs que j'étudie et de les évaluer pour proposer des pistes d'amélioration, je me dois tout de même de m'efforcer de préserver l'intégrité de cet écosystème social. C'est nécessairement délicat, puisque le but avoué d'emblée est d'élaborer des propositions d'intervention, mais qu'il faut aussi éviter les bouleversements inattendus qui peuvent découler de la présence du chercheur sur le terrain et du passage de l'implicite à l'explicite dans certains rapports sociaux. Quel que soit l'équilibre qui caractérise le milieu, je considère comme chercheur devoir intervenir avec autant de délicatesse que possible. Une unité au sein d'un hôpital n'est pas nécessairement entièrement harmonieuse, loin de là. Elle peut aussi être le théâtre de débats récurrents, d'inimitiés et de rivalités, et l'intrusion d'un chercheur peut avoir un impact négatif sur ces dynamiques. 
Cette idée est parfaitement illustrée par l'évocation, dans "Parlons d'œufs », de l'arrivée d'une caméra de sécurité surveillant l'extérieur du Musée d'art contemporain de Montréal dans la foulée de la manœuvre clandestine d'Arkadi Lavoie Lachapelle et d'Audrey Racicot. Laissons ouverte la question de savoir s'il est désirable d'exercer une surveillance accrue sur l'extérieur du Musée, avec tout ce que cela peut entrainer comme conséquences pour les habitués du quartier tels que, par exemple, les gens de la rue. Toutefois, même s'il n'est pas clair si la manœuvre a directement causé l'installation de la caméra ou si cette corrélation est entièrement fortuite, cela démontre au moins que les conséquences de nos interactions avec le monde sont susceptibles de nous échapper en tout ou en partie. Ainsi, en effectuant une manœuvre que l'on conçoit comme tournée vers l'intérieur du musée en tant qu'institution culturelle, vers les conceptions multiples de l'art, des artistes et de la conservation de ses acteurs, on peut éventuellement avoir un impact sur l'extérieur du musée, sur sa place dans la ville, sur la façon dont son caractère d'espace public s'incarne ainsi que les limites de celui-ci.

Est-ce une raison pour faire preuve de plus de retenue, tant pour les chercheurs que pour les artistes? Je suis tenté de dire : retenue non, prudence oui. Autant les chercheurs que les artistes peuvent, s'ils en ont le désir et la capacité, continuer à essayer de repousser les limites de notre compréhension du monde et s'efforcer d'œuvrer pour la justice. L'art et la recherche peuvent être arrimés à des objectifs normatifs et prendre part à des pratiques et à des processus sociaux dans l'espoir d'assouplir le statu quo et de dégager des leviers d'intervention. À cet égard, il faut demeurer vigilant pour que l'éthique ne bascule pas dans la gestion du risque juridique et réputationnel et devienne un dispositif de contrôle institutionnel.

Cela dit, les chercheurs et les artistes doivent assumer la responsabilité de leurs interventions dans le monde. L'art, surtout s'il se fait action et intervient directement, n'est pas une sphère distincte de la vie sociale et, s'il est régi par certaines règles ayant émergé des interactions entre ses acteurs et qui lui sont propres (et dont la remise en question constitue un des moteurs), il n'en demeure pas moins que les principes fondamentaux de l'éthique tels que le respect pour les personnes ou la protection de l'environnement l'interpellent. Ainsi, l'œuvre crée un monde en elle-même, mais elle est néanmoins conçue comme s'insérant dans le monde. Par exemple, un aspect resté dans l'ombre de " 1600 œufs » et de "Parlons d'œufs! » et dont j'ai eu la chance de discuter avec Arkadi Lavoie Lachapelle est le souci éthique qui a guidé toute la démarche. En effet, tout d'abord, la finalité alimentaire justifiant (du moins en partie) la production industrielle d'œufs a été respectée dans le cadre du projet, la technique utilisée pour évider les œufs visant entre autres à s'assurer que les œufs demeuraient comestibles. Ensuite, une partie de ses œufs ont été consommés par les artistes et leurs proches et l'autre partie a été remise à La Maison du Père, un refuge montréalais pour personnes itinérantes. De même, lorsque la manœuvre clandestine accompagnant " Parlons d'œufs! ", de nouveau réalisée avec la collaboration d'Audrey Racicot, a été contestée par un autre artiste selon qui elle mettait en péril l'intégrité de l'œuvre qu'il exposait dans une des salles de la galerie devant l'entrée de laquelle le duo a installé sa matrice d'œufs remplis de paillettes multicolores ; la décision fut prise d'y mettre un terme.

Finalement, cette responsabilité devrait aussi nous entrainer sur la piste d'une réflexion sur le lien entre le produit fini, qu'il s'agisse d'une œuvre artistique ou d'un article scientifique, et les individus et les milieux de vie qui, par leur participation spontanée ou orchestrée ou par leurs confidences, y ont participé. Je ne veux pas diminuer l'immense travail que représente une étude empirique qualitative ou la réalisation d'une manœuvre en art-action. En ce qui concerne le premier cas de figure, je sais que j'y consacre plusieurs heures chaque semaine, et ce depuis plusieurs années déjà. Par contre, force est d'admettre qu'il ne me serait pas même possible d'investir tout ce temps, cette énergie et cette réflexion si ce n'était du concours précieux des participants à mon étude. Individuellement, bien peu d'entre eux seraient en mesure de mener à bien pareil projet, voire d'anticiper et de déduire à partir de leur propre expérience, mes conclusions et mes recommandations. Ainsi, autant le chercheur que les participants sont des conditions nécessaires à la réalisation du projet, mais ni l'un ni les autres n'est une condition suffisante. 
De cette interdépendance résulte une revendication légitime des participants vis-à-vis du projet de recherche ou de l'œuvre. Le droit du chercheur se trouve en quelque sorte grevé par ceux des participants. Ce dernier ne peut donc pas se concevoir en monarque absolu vis-à-vis des données recueillies ou du produit final. Au minimum, cela vient apporter support à l'obligation éthique du chercheur de rendre ses résultats accessibles aux individus et aux communautés avec lesquelles il a travaillé. Notons ici que rendre des résultats accessibles implique vraisemblablement plus que d'envoyer des tirés à part d'articles scientifiques ou une copie d'une volumineuse thèse de doctorat. Je pense plutôt à un exercice de vulgarisation permettant de rendre manifeste la pertinence de la recherche pour le milieu d'où elle a émergé. La question se pose ensuite de savoir si les participants ne devraient pas avoir une plus grande influence sur la détermination des objectifs de la recherche et sur sa réalisation. II est intéressant de noter que dans l'énoncé de politique en éthique de la recherche des trois conseils de recherche du Canada [11], la participation de la communauté a été identifiée comme une dimension éthique importante de la recherche impliquant les Autochtones (chapitre 9), mais occupe une place beaucoup moins importante dans d'autres contextes. Or, il y a lieu de se demander si cette distinction est légitime.

Si l'on revient sur l'œuvre réalisée par Arkadi Lavoie Lachapelle dans le cadre de ce projet et sur la façon dont elle a choisi de la présenter, on peut constater d'emblée qu'elle est soucieuse de cette dimension d'interdépendance en cela que dans le cartel accompagnant l'œuvre, on retrouve une liste détaillée de collaborateurs, de l'auteure de la prise de vue, en passant par l'encadreur jusqu'au bioéthicien. En partageant la scène avec ses collaborateurs, l'artiste se détache de l'idéal romantique du génie créant à partir de rien et partant, donne une image plus sociale qu'individuelle de son travail et de son œuvre.

\section{Conclusion}

Ce qui précède ne représente en somme que les balbutiements de la réflexion et du dialogue que j'ai entamé sur l'art et la bioéthique en collaboration avec Arkadi Lavoie Lachapelle. D'autres avenues restent à être explorées et les méthodes pour se faire restent à être découvertes ou plus vraisemblablement à être inventées. En effet, pour aller plus en avant avec cette collaboration, il faut désormais essayer de repenser le sens du "+ » dans l'équation : "Art + Bioéthique ». S'agit-il d'une simple juxtaposition? Avons-nous affaire à une tautologie où les deux termes sortent indemnes de la rencontre $($ Art + Bioéthique $=$ Art + Bioéthique $)$ ou est-ce que le résultat représente plus que la somme des parties?

Avant même de m'engager dans ce projet, j'étais persuadé du potentiel de la rencontre entre l'art et la bioéthique, ayant déjà utilisé des romans et des récits littéraires dans le cadre d'un cours de droit que je donnais et m'étant aussi déjà penché sur une œuvre artistique, dans ce cas une pièce de théâtre, avec une lunette éthique [12]. Cependant, grâce à ma participation à ce projet, j'ai eu l'occasion d'être associé de près à la réflexion entourant la conception et la réalisation d'une œuvre d'art, réfléchissant avec Arkadi Lavoie Lachapelle aux implications artistiques, éthiques, politiques et économiques des choix qui se sont présentés à elle. En ce sens, ma position par rapport à l'équation "Art + Bioéthique " a déjà changé et avec elle ma perspective. Au moment d'écrire ces lignes, nous préparons une conférence qui sera présentée à la galerie Espace Projet en mars 2016 dans le cadre de l'exposition. Déjà, cette nouvelle étape de notre collaboration nous permet d'approfondir notre dialogue et de jeter les bases d'un langage commun. En effet, après avoir invité le regard de l'autre dans le développement en parallèle de produits typiques de nos champs de pratique respectifs une œuvre d'art d'une part et un texte d'autre part, nous passons à une autre étape en explorant une nouvelle modalité de l'équation dans le cadre de cette conférence. D'autres collaborations pourraient aussi suivre. Une chose est certaine, ce n'est pas la fin de l'expérience. 


\section{Références}

1. Cover RM. Foreword: Nomos and narrative. Harvard Law Review. 1983;97:4.

2. Rodriguez c. Colombie-Britannique (Procureur général). RCS. 1993. p. 519.

3. Carter c. Canada (Procureur général). RCS. 2015. p. 331.

4. Melissaris E. Ubiquitous Law Legal Theory and the Space for Legal Pluralism. Farnham, UK; Burlington, VT: Ashgate Pub.; 2009.

5. Davies R, Ives J, Dunn M. A systematic review of empirical bioethics methodologies. BMC Medical Ethics. 7 mars 2015;16(1):15.

6. Guillemette F, Luckerhoff J. Méthodologie de la théorisation enracinée: fondements, procédures et usages. Québec: Presses de l'Université du Québec; 2012.

7. Ives J. 'Encounters with experience': empirical bioethics and the future. Health Care Analysis. 2008;16(1):1-6.

8. Lavoie Lachapelle, A. «1600 oeufs », manoeuvre clandestine devant le Musée dart contemporain de Montréal, 17 décembre 2010, oeufs, paillettes, plomb, en collaboration avec Audrey Racicot.

9. Lavoie Lachapelle, A. «Parlons d'oeufs! », 2010-2015, couleur, son, vidéo, 15min.06s +manoeuvre clandestine devant la Galerie de l'UQAM le 8 janvier 2015 avec la collaboration d'Audrey Racicot.

10. Lavoie Lachapelle, A. « Jour de fête », manoeuvre dans le quartier des Affaires de Montréal, Canada, 13 avril 2011.

11. Groupe consultatif interagences en éthique de la recherche. Énoncé de politique des trois Conseils : Éthique de la recherche avec des êtres humains. Décembre 2014

12. Laflamme E, Ménard J-F. Hyperréalisme des sentiments. Spirale Arts• Lettres• Sciences Humaines. 2011;(237):78-9. 